Check for updates

Cite this: RSC Adv., 2017, 7, 50072

\title{
Molecular recognition of pyruvic acid and folic acid in whole blood
}

\author{
Raluca-Ioana Stefan-van Staden, (D) *ab Amalia Gabriela Diaconeasa, ${ }^{\mathrm{b}}$ \\ Livia Alexandra Gugoasa, ${ }^{a}$ Marcela-Corina Rosu ${ }^{c}$ and Stela Pruneanuc
}

Pyruvic and folic acids need to be monitored in both young and old persons, not only to assess the ageing state, but also to diagnose illnesses at an early stage, e.g., diabetes. Therefore, there is a need to develop highly reliable tools and methods of analysis for their determination in biological fluids (e.g., whole blood samples). Three new stochastic microsensors based on the physical immobilization of $\alpha, \beta$, and $\gamma$ cyclodextrins (CD) in the paste of a $\mathrm{TiO}_{2} \mathrm{Pt}$-graphene nanocomposite $\left(\mathrm{TiO}_{2} \mathrm{Pt} / \mathrm{rGO}\right)$ were prepared; the novelty of the stochastic sensors used for the assay of the biomolecules from biological fluids is given by the utilization of $\mathrm{TiO}_{2} \mathrm{Pt}$-graphene nanocomposite as the matrix; this matrix supported the CDs' channels used for stochastic sensing, better than other materials. The proposed microsensors are able to recognize and determine pyruvate and folate in whole blood samples at very low concentrations. The recovery tests performed for the assay of pyruvate and folate in whole blood samples proved that they can be assayed with a recovery higher than $98.00 \%$ and an RSD value lower than $1.00 \%$.

Received 21st August 2017

Accepted 16th October 2017

DOI: 10.1039/c7ra09260a

rsc.li/rsc-advances
Stochastic sensors are well known for their capacity of performing both qualitative and quantitative types of reliable analysis. They can be used for molecular recognition of different biomarkers due to their working principle: only one molecule gets through the channel at a time. The mechanism of molecular recognition and quantitative assessment using stochastic sensors is simple: the molecule gets in the channel the time needed to go inside the channel being known as the signature of the marker (measured using the $t_{\text {off value); at this }}$ stage, the channel is blocked and the current drops to zero; the second stage, used for quantitative assessment of the molecules, is assessed through the time (known as $t_{\text {on }}$ ) when processes like binding of the molecule on the channel and redox processes take place inside the channel. ${ }^{11,12}$ This facilitates the multianalyte assay in biological matrices; the order of entering the channel being given by the size, geometry, conformation, unfolding capacity of the molecules and their velocity of entering the channel.

The design of stochastic sensors is based on the utilization of nanostructured materials such as graphene based materials. Graphene-modified detection systems represent a novel approach in the construction of electrochemical sensors, since such a material has tunability as well as novel electrical and catalytic properties that emerge at this scale. The results are tremendous in terms of sensitivity, selectivity and versatility. ${ }^{\mathbf{1 3}}$

This paper describes for the first time the design, characterization and validation of three stochastic microsensors based on the immobilization of $\alpha, \beta$, and $\gamma$-cyclodextrins (CD) in a paste of a $\mathrm{TiO}_{2} \mathrm{Pt}$ graphene nanocomposite $\left(\mathrm{TiO}_{2} \mathrm{Pt} / \mathrm{rGO}\right)$, for molecular recognition of pyruvic acid and folic acid in whole 
blood samples. The main advantage of using the graphene nanocomposites versus other materials employed for the stochastic sensors' design is that its nanostructure can support the CD channel necessary for stochastic sensing better.

\section{Experimental}

\section{Materials and reagents}

Pyruvic acid, folic acid, and $\alpha, \beta$, and $\gamma$-cyclodextrins (CD) were purchased from Sigma Aldrich (Milwaukee, USA) and paraffin oil $\left(d_{4}^{20}, 0.86 \mathrm{~g} \mathrm{~cm}^{-3}\right)$ from Fluka (Buchs, Switzerland).

Monosodium and disodium phosphates were used for the preparation of a $0.1 \mathrm{~mol} \mathrm{~L}^{-1}$ phosphate buffer saline (PBS) of $\mathrm{pH}$ 7.4. Deionized water obtained from a Millipore Direct-Q 3 System (Molsheim, France) was used for the preparation of all solutions. All standard solutions were prepared using distilled water and the buffer solution $(\mathrm{pH}=7.4)$ in the ratio distilled water: buffer solution $=1: 1(\mathrm{v} / \mathrm{v})$. The serial dilution technique was used for the preparation of solutions of different concentrations of pyruvic and folic acids (from $1 \mathrm{mmol} \mathrm{L}^{-1}$ down to 1 fmol $\mathrm{L}^{-1}$ ). The solutions were stored in the fridge at $2-8{ }^{\circ} \mathrm{C}$. All chemicals were of analytical grade. The preparation of the $\mathrm{TiO}_{2} \mathrm{Pt} / \mathrm{rGO}$ nanocomposite was described in detail in our recently published paper. ${ }^{\mathbf{1 4}}$

\section{Design of the stochastic microsensors based on $\mathrm{TiO}_{2} \mathrm{Pt} / \mathrm{rGO}$}

Paraffin oil was added to the $\mathrm{TiO}_{2} \mathrm{Pt} / \mathrm{rGO}$ powder until a homogenous paste was formed. To each $10 \mathrm{mg}$ paste, $5 \mu \mathrm{L}$ of modifier $\left(10^{-3} \mathrm{~mol} \mathrm{~L}^{-1}\right.$ solution of either $\alpha, \beta$, or $\gamma$-cyclodextrins) was added to give the modified paste. Each modified paste (obtained by a physical mixture of graphene nanocomposite, paraffin oil and CD solution) was inserted in a plastic tip with a diameter of $250 \mu \mathrm{m}$ for the active site. The electrical contact was obtained using an Ag wire. When not in use, the stochastic microsensors were stored at room temperature, in a dry place.

\section{Apparatus and methods}

All measurements were performed with an AUTOLAB/PGSTAT 12 (Metrohm) system connected to a computer. A threeelectrode electrochemical cell system was employed. An Ag/ $\mathrm{AgCl}$ electrode (with $0.1 \mathrm{~mol} \mathrm{~L}^{-1} \mathrm{KCl}$ solution) served as the reference electrode in the cell while a Pt wire served as the auxiliary electrode.

A stochastic mode was used for the measurement of the qualitative parameter $\left(t_{\mathrm{off}}\right)$ and the quantitative parameter $\left(t_{\mathrm{on}}\right)$, at a constant potential (125 $\mathrm{mV}$ versus $\mathrm{Ag} / \mathrm{AgCl})$ and was used for both molecular recognition (qualitative analysis) and quantitative analysis of pyruvic and folic acids (Fig. 1). The signatures of the acids (the values of $t_{\text {off }}$ shown in Table 1) were used to identify pyruvic acid and folic acid in each diagram; after their identification, the values of $t_{\text {on }}$ were read and used for the determination of the concentrations of pyruvic and folic acids in whole blood samples, using the equation $1 / t_{\text {on }}=a+b x_{\text {conc }}$.

The morphology of the $\mathrm{TiO}_{2} \mathrm{Pt} / \mathrm{rGO}$ composite was examined with a transmission electron microscope (H-7650 Hitachi Japan) operated at $120 \mathrm{kV}$. Elemental analysis was performed
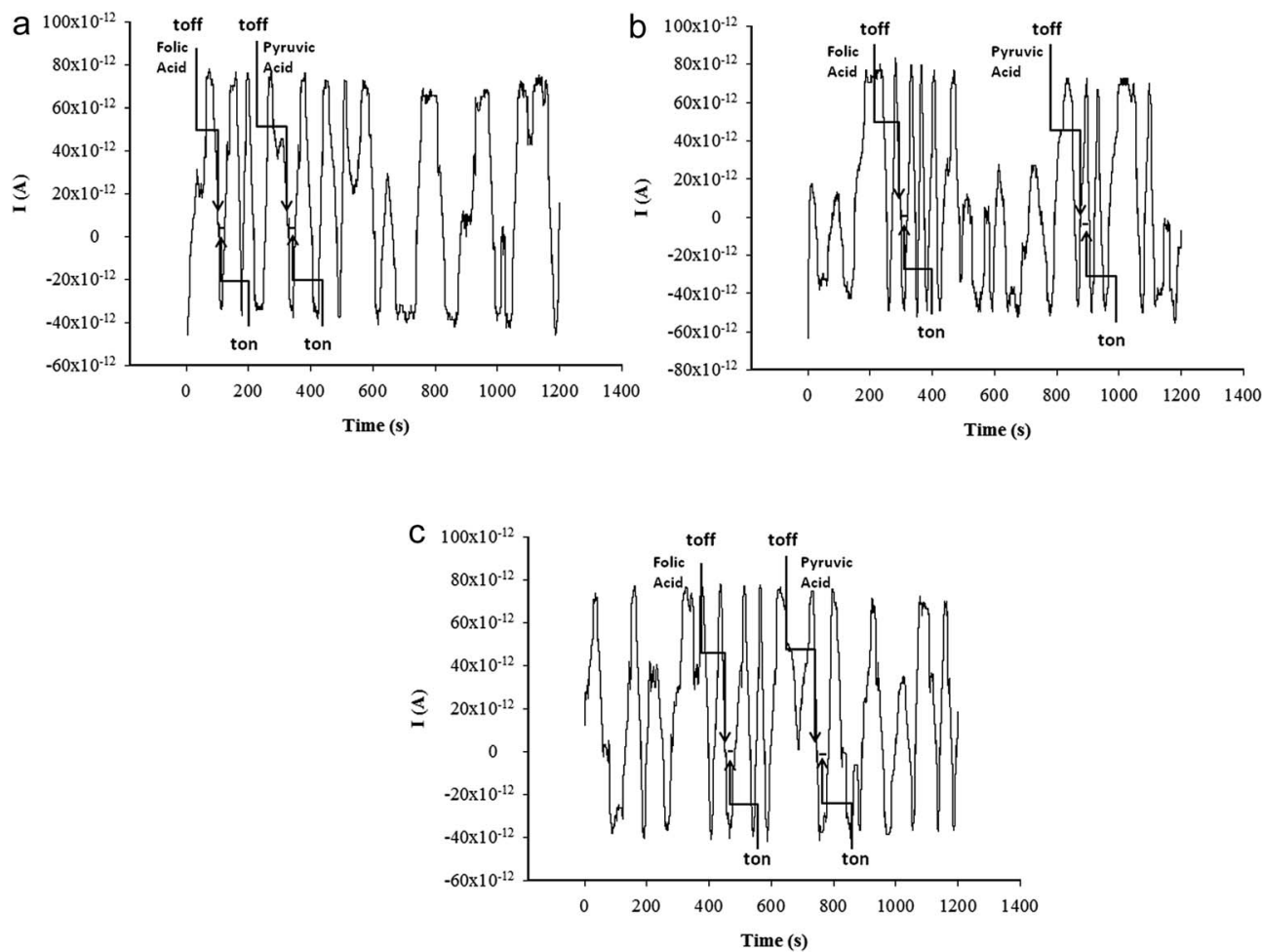

Fig. 1 Diagrams obtained for the screening test of whole blood using the sensors based on Pt-TiO $2 / \mathrm{rGO}$ paste and (a) $\alpha$-CD, (b) $\beta$-CD, and (c) $\gamma$ CD. 
Table 1 Response characteristics of stochastic sensors used for the assay of pyruvic and folic acids

Microsensors based on $\mathrm{Pt}-\mathrm{TiO}_{2} /$ rGO paste, modified with
Calibration equation ${ }^{a}$ and correlation coefficient $(r)$
Linear concentration range $\left(\mathrm{mol} \mathrm{L}^{-1}\right)$
Limit of determination $\left(\mathrm{mol} \mathrm{L}^{-1}\right)$

Pyruvic acid

$\alpha$-CD

$\beta-\mathrm{CD}$

$\gamma-\mathrm{CD}$
$1 / t_{\text {on }}=0.04+3.86 \times 10^{6} \times C, r=0.9995$

$1 / t_{\mathrm{on}}=0.05+3.47 \times 10^{5} \times C, r=0.9999$

$1 / t_{\text {on }}=0.05+5.42 \times 10^{6} \times C, r=0.9999$

$\begin{array}{ll}1.0 \times 10^{-10}-1.0 \times 10^{-8} & 1.8 \\ 1.0 \times 10^{-9}-1.12 \times 10^{-7} & 1.2 \\ 1.00 \times 10^{-10}-1.00 \times 10^{-8} & 1.2\end{array}$

1.8

$\begin{array}{ll}1.0 \times 10^{-1.12} \times 10^{-1} & 1.2 \\ 1.00 \times 10^{-10}-1.00 \times 10^{-8} & 1.2\end{array}$
$3.86 \times 10^{6}$

$3.47 \times 10^{5}$

$5.42 \times 10^{6}$

$1.0 \times 10^{-10}$

$1.0 \times 10^{-9}$

$1.00 \times 10^{-10}$

\section{Folic acid}

$\alpha-\mathrm{CD}$

$\beta-\mathrm{CD}$

$1 / t_{\text {on }}=0.05+3.44 \times 10^{7} \times C, r=0.9999$

$1 / t_{\text {on }}=0.02+3.21 \times 10^{7} \times C, r=0.9999$

$1.00 \times 10^{-11}-1.00 \times 10^{-9} \quad 2.5$

$1.00 \times 10^{-11}-1.00 \times 10^{-9} \quad 1.5$

$\gamma$-CD

$1 / t_{\text {on }}=0.03+2.24 \times 10^{4} \times C, r=0.9999$

$1.00 \times 10^{-11}-1.00 \times 10^{-6}$

2.0

$3.44 \times 10^{7}$
$3.21 \times 10^{7}$
$2.24 \times 10^{4}$

$1.00 \times 10^{-11}$

$1.00 \times 10^{-11}$

$a_{<1 / t_{\mathrm{on}}>}=\mathrm{s}^{-1},<C>=\mathrm{mol} \mathrm{L}^{-1}$.

with EDS, the accessory of TEM. The sample was dispersed in ethanol, dropped on a copper grid (200 mesh) and dried at room temperature for several minutes.

\section{Samples}

16 whole blood samples were collected from patients from the University Hospital in Bucharest (Ethics committee approval no. 75/2015). Informed consent was obtained from all patients. The samples were screened using the multimode sensors, without any sample treatment.

\section{Results and discussions}

\section{TEM-EDS analysis of $\mathrm{TiO}_{2} \mathrm{Pt} / \mathrm{rGO}$ composite}

A representative TEM image of $\mathrm{TiO}_{2} \mathrm{Pt} / \mathrm{rGO}$ composite can be seen in Fig. 2a. The graphene sheets are very thin, being composed of 2-3 layers, and are decorated with $\mathrm{TiO}_{2} \mathrm{Pt}$ nanoparticles (size below $50 \mathrm{~nm}$ ). The corrugated graphene surface as well as the presence of $\mathrm{TiO}_{2} \mathrm{Pt}$ nanoparticles leads to an increase in the active surface area of the paste electrode. The elemental maps of Ti, Pt and $\mathrm{O}$ in the sample can be seen in Fig. 2b-e, respectively. A low number of Pt nanoparticles was identified by EDS mapping (Fig. 2d) due to the low

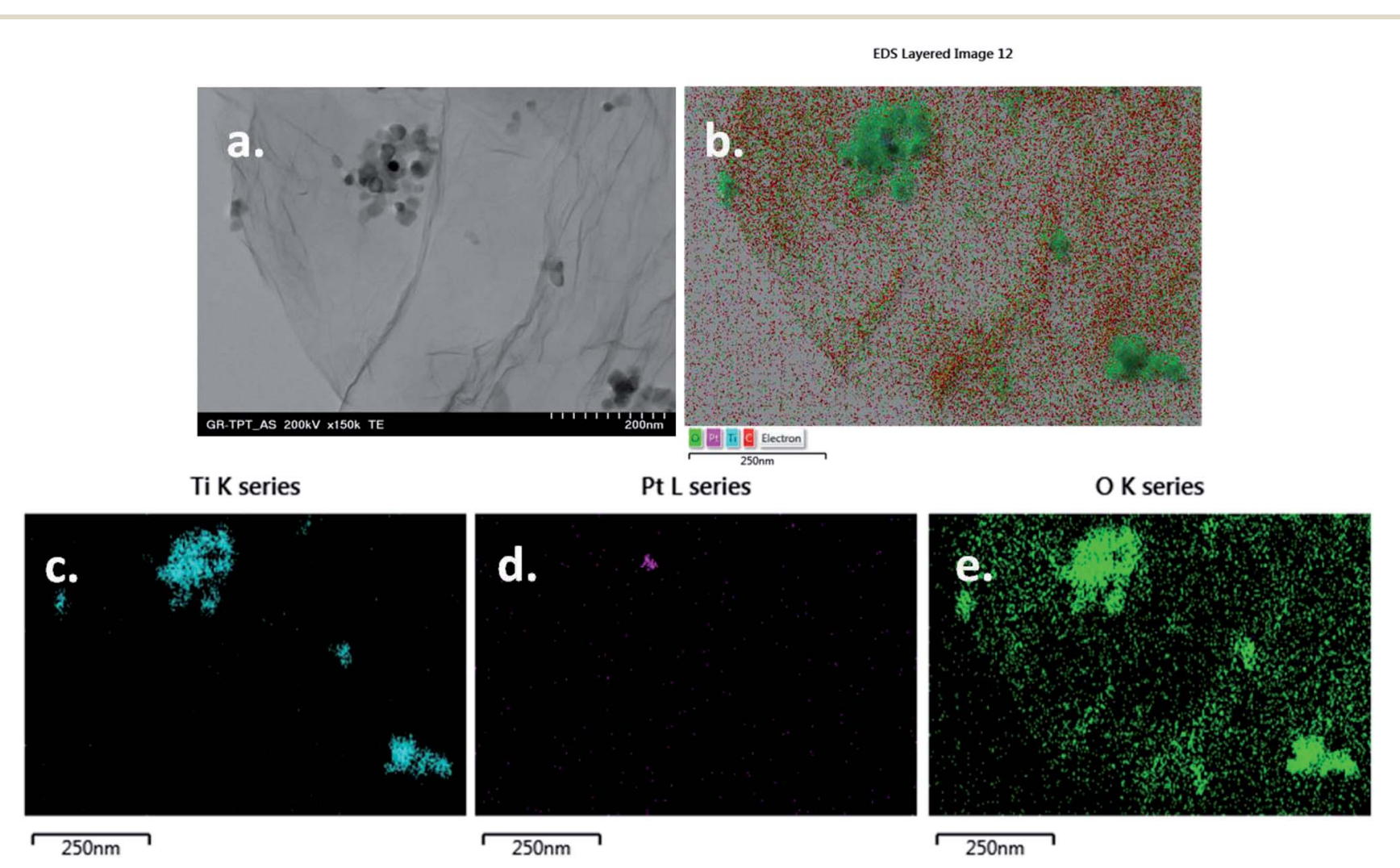

Fig. 2 Representative TEM image of $\mathrm{TiO}_{2} \mathrm{Pt} / \mathrm{rGO}$ sample (a) scale bar $200 \mathrm{~nm}$; elemental maps of Ti, Pt and O in the same sample (b-e). 
concentration of hexachloroplatinic acid used for the synthesis of $\mathrm{TiO}_{2} \mathrm{Pt}$ composite.

\section{Response characteristics of the stochastic microsensors used for the assay of pyruvic and folic acids}

Stochastic response relies on the conductivity of channels when the analyte is going through it: under an applied potential, the molecule of the analyte is extracted from the solution to the membrane-solution interface. Molecular recognition of the analyte takes place in 2 stages. In the first stage, the molecule of the analyte blocks the entrance in the pore (the current drops to zero) for a certain period of time, which is called $t_{\text {off }}$ and represents the qualitative parameter of the analyte. In the second stage, the molecule binds to the pore wall and the redox processes take place. The binding time is called $t_{\text {on }}$ and represents the quantitative parameter.

According to the stochastic mode described above, ten diagrams for each concentration were obtained separately for folic and pyruvic acids. In these diagrams, only the peaks related to the markers (folic acid or pyruvic acid) showed a change in their width, and therefore they could be easily identified, and the values of the $t_{\text {off }}$ (signatures of the pyruvic and folic acids) were determined. The $t_{\text {on }}$ values were read for each signature of the pyruvic and folic acids, and they were further used for the statistic evaluation of all response characteristics and the determination of the equations of calibration for each acid when a different stochastic sensor was used. Table 1 presents the response characteristics of the stochastic microsensors designed for the assay of pyruvic and folic acids. The signatures of pyruvic acid and folic acid ( $t_{\text {off }}$ values) are different for the same microsensor; accordingly, these acids can be simultaneously determined from the same sample, with the proposed microsensor. Calibration plots are shown in Fig. 3 and 4.

The largest linear concentration range for the assay of folic acid was obtained with the microsensor based on $\gamma$-CD. The best sensitivities for the assay of pyruvic acid were obtained with the microsensors based on $\alpha$ and $\gamma$-CD (both have the same order of magnitude), while for the assay of folic acid, the best sensitivity was obtained when the microsensor based on $\alpha$-CD was employed. The limits of determination recorded for folic acid were the same for the three microsensors, while for the assay of pyruvic acid, the lowest values were obtained when the microsensors based on $\alpha$ - and $\gamma$-CD were used. The sensor of choice for simultaneous assay of pyruvic and folic acids was the one based on $\alpha$-CD. The proposed stochastic sensors covered the ranges for folic acid $\left(4.5-45.3 \mathrm{nmol} \mathrm{L}^{-1}\right)$ and for pyruvic acid $\left(8-160 \mathrm{nmol} \mathrm{L}^{-1}\right)$ for healthy patients, as well as lower and higher ranges of concentrations for the assay of the pyruvic and folic acids in whole blood samples for ill patients.

The reliability of the design was determined for each sensor according to the following procedure: three modified graphene pastes were made from each $\mathrm{CD}$ and the graphene nanocomposite, and the response characteristics were determined for all of these sensors and compared; for each CD based stochastic sensor, the value of the sensitivity (the slope of the calibration graph) varied with less than $1.00 \%$. For each sensor, the response characteristics were determined every day for one month; in this period, the sensitivity (the slope of the calibration graph) of each sensor varied less than $1.00 \%$, and therefore one can say that the proposed stochastic sensors are stable over time. The reliability and
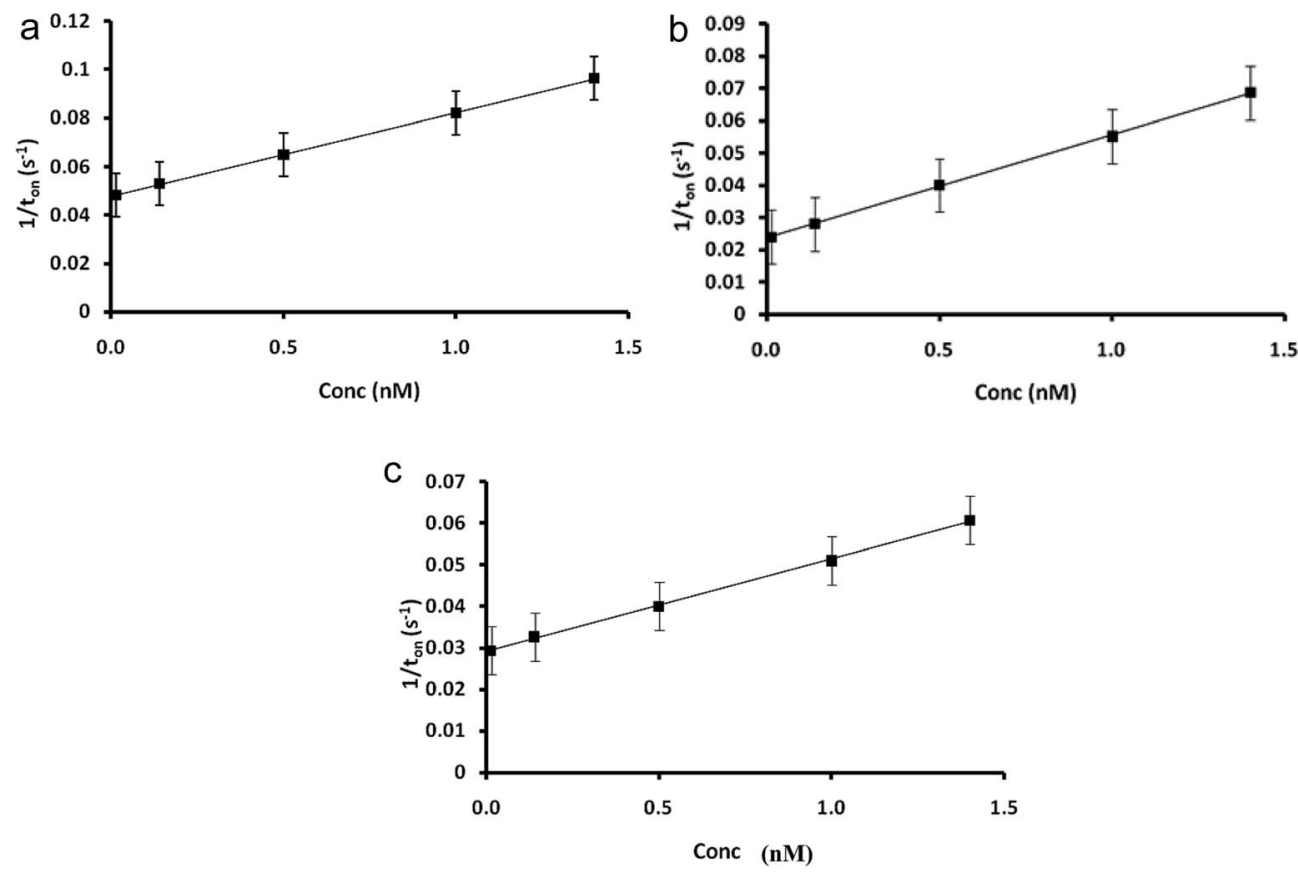

Fig. 3 Calibration plots for folic acid obtained for the sensors based on Pt-TiO $2 / \mathrm{rGO}$ paste and (a) $\alpha-C D$, (b) $\beta-C D$, and (c) $\gamma-C D$. 

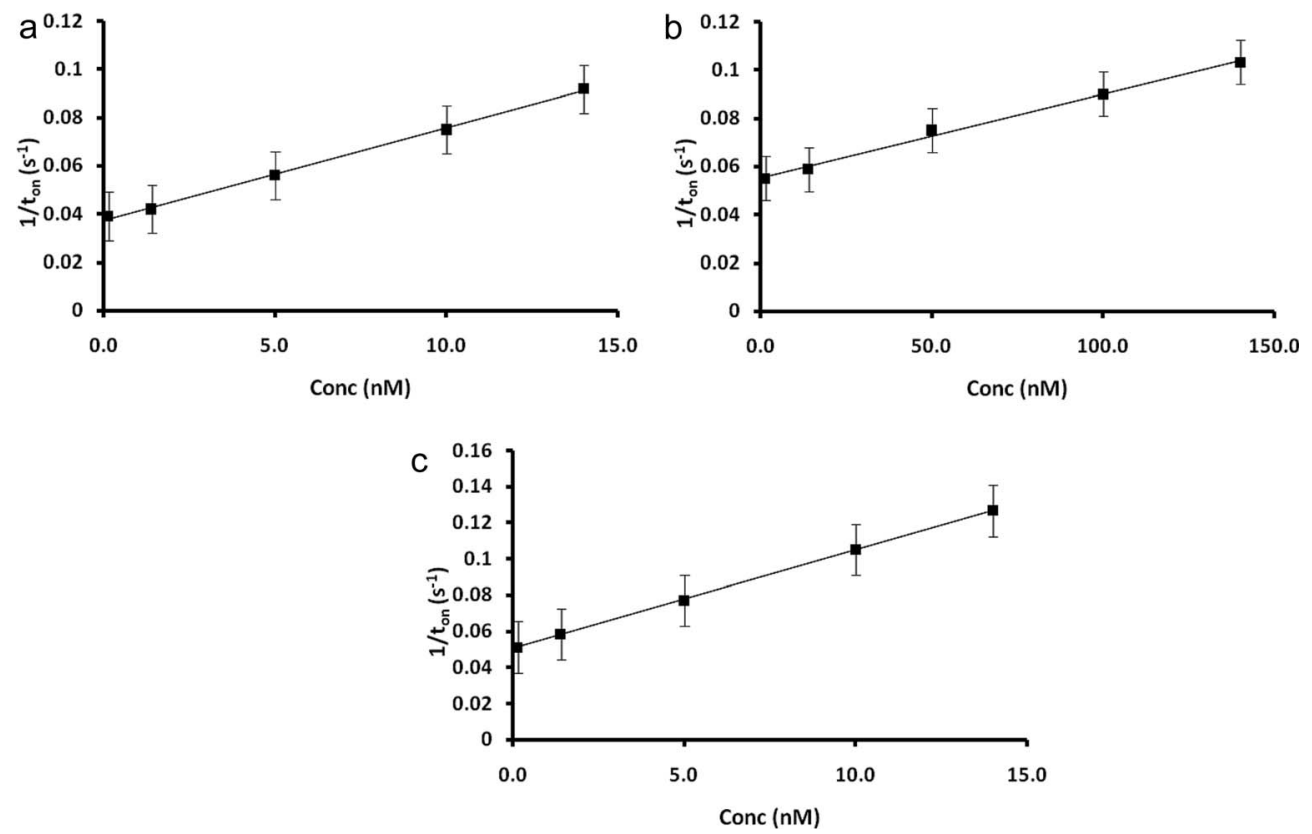

Fig. 4 Calibration plots for pyruvic acid obtained for the sensors based on Pt-TiO $2 / r G O$ paste and (a) $\alpha-C D$, (b) $\beta-C D$, and (c) $\gamma-C D$.

Table 2 Recovery tests of pyruvic and folic acids in whole blood samples $(N=10)$

\begin{tabular}{lll}
\hline & \multicolumn{2}{l}{$\%$, recovery of } \\
\cline { 2 - 3 } $\begin{array}{ll}\text { Microsensor based on } \\
\text { Pt-TiO }_{2} / \text { rGO paste, modified with }\end{array}$ & Pyruvic acid & Folic acid \\
\hline$\alpha-C D$ & $99.98 \pm 0.02$ & $99.95 \pm 0.01$ \\
$\beta$-CD & $98.89 \pm 0.05$ & $98.72 \pm 0.03$ \\
$\gamma$-CD & $98.20 \pm 0.08$ & $98.35 \pm 0.07$
\end{tabular}

stability in time were also due to the graphene nanocomposite material used as the matrix for physical immobilization of different CDs, the combination being a novelty in the field of stochastic sensors.

Interferences were checked versus neurotransmitters like dopamine, epinephrine, and norepinephrine. Different signatures ( $t_{\text {off }}$ values) were obtained for the proposed interferences, and accordingly the proposed microsensors are deduced to be selective. This may have happened because no cross-reactivity takes place during utilisation of this method, the mechanism of stochastic sensors' response being based on single events taking place at a time - each molecule gets inside the channel in an order given by size, geometry, conformation, and velocity of unfolding (for 2D/3D structures), and velocity of getting into the channel, all these parameters being independent on the nature and complexity of the matrix from where the main marker/ substance is determined. This also explains the different values obtained for the signatures of different markers/ substances.

\section{Analytical applications}

The response characteristics of the proposed microsensors (Table 1) show that folic and pyruvic acids can be simultaneously determined using the proposed microsensors from whole blood samples (different values for their signatures were determined).

Recovery tests were performed as follows: for different whole blood samples, the concentrations of folic and pyruvic acids were determined and then different volumes of known concentrations of folic and pyruvic acids were added. The differences between the found amount and initial amount of folic and pyruvic acid, respectively, were compared with the added amounts of folic and pyruvic acids, and expressed in percentage recovery. The recovery tests (Table 2) performed versus standard methods (spectrometric methods of analysis) show recoveries higher than $98.00 \%$ for both acids using the proposed microsensors and RSD values less than $1.00 \%$, proving high accuracy and precision of the measurements.

Whole blood samples were collected from 16 patients for the validation of the proposed stochastic microsensors. The whole blood samples were placed in the measurement cell, and the diagrams were recorded (Fig. 1); pyruvic acid and folic acid were identified in the diagrams based on their signatures (Table 1), and after that, $t_{\text {on }}$ values (Fig. 1 ) were measured and used for the quantitative analysis of the acids in whole blood samples. The results presented in Table 3 show good agreement between the results recorded with the proposed stochastic sensors, and standard methods (commercial kits were used) and low values for the RSD measurements performed with stochastic sensors. Accordingly, the concentrations of folic and pyruvic acids can be reliably determined from whole blood samples. 
Table 3 Determination of pyruvic and folic acids in whole blood samples $(N=3)$

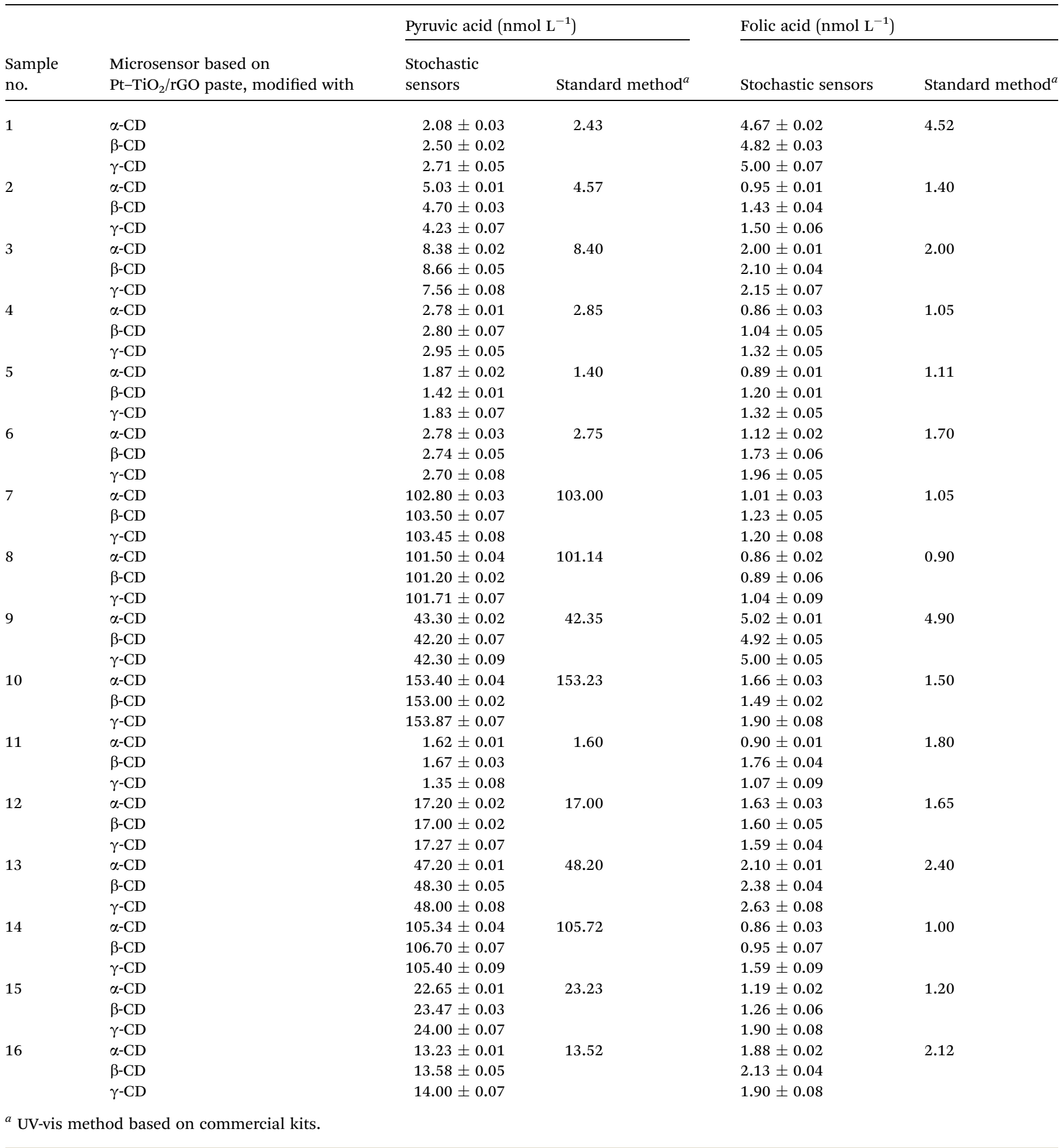

\section{Conclusions}

In this study, we proposed three stochastic microsensors based on the immobilization of $\alpha, \beta$, and $\gamma$-cyclodextrins (CD) in a paste of $\mathrm{TiO}_{2} \mathrm{Pt}$ graphene nanocomposite $\left(\mathrm{TiO}_{2} \mathrm{Pt} / \mathrm{rGO}\right)$. The novelty is in the utilization of a graphene nanocomposite for the immobilisation of different CDs. The best results for the simultaneous assay of folic and pyruvic acids were obtained when $\alpha$-CD was used for the design of the microsensor. The proposed method is simple, fast, and accurate compared with those previously proposed and can be used for the screening of whole blood samples, as taken from the patients. 


\section{Conflicts of interest}

The authors declared no conflicts of interest.

\section{Acknowledgements}

This work was supported by UEFISCDI, PNCDI III framework, PCE project, contract no. 46/2017. The authors want to thank Alin Sebastian Porav for performing the TEM investigation.

\section{References}

1 E. H. Reynolds, Folic acid, ageing, depression, and dementia, $B M J, 2002$, 324, 1512-1515.

2 J. Mehla, B. C. Chauhan and N. B. Chauhan, Experimental induction of type 2 diabetes in aging-accelerated mice triggered Alzheimer-like pathology and memory deficits, $J$. Alzheimer's Dis., 2014, 39, 145-162.

3 R. Iyer and S. K. Tomar, Determination of folat/folic acid level in milk by microbiological assay, immunoassay, and high performence liquid chromatography, J. Dairy Res., 2013, 80, 233-239.

4 A. Zhu, R. Romero and H. R. Petty, A sensitive fluorimetric assay for pyruvate, Anal. Biochem., 2010, 396, 146-151.

5 G. Xuan, X. Lu, J. Wang, H. Lin and H. Liu, Determination of pyruvic acid concentration using a bioluminescence system from Photobacterium leiognathi, Photochem. Photobiol. Sci, 2015, 14, 1163-1168.

6 A. G. Hadjivassiliou and S. V. Rieder, The enzymatic assay of pyruvic and lactic acids. A definitive procedure, Clin. Chim. Acta, 1968, 19, 357-361.
7 G. E. Anthon and D. M. Barett, Modified method for the determination of pyruvic acid with dinitrophenylhydrazine in the assessment of onion pungency, J. Sci. Food Agric., 2003, 83, 1210-1213.

8 R. Amidzic, J. Brboric, O. Cudina and S. Vladimirov, RPHPLC determination of vitamins B1, B3, B6, folic acid and B12 in multivitamin tablets, J. Serb. Chem. Soc., 2005, 70, 1229-1235.

9 V. D. Reif, J. T. Reamer and L. T. Grady, Chromatographic assay of folic acid, J. Pharm. Sci., 1977, 66, 1112-1116.

10 A. Lebiedzinska, M. Dabrowska, P. Szefer and M. Marszall, High-performance liquid chromatography method for the determination of folic acid in fortified food products, Toxicol. Mech. Methods, 2008, 18, 463-467.

11 R. I. Stefan-van Staden, I. R. Comnea-Stancu, C. C. SurduBob and M. Badulescu, Nanostructured materials detect epidermal growth factor receptor, neuron specific enolase and carcinoembryonic antigen, Nanoscale, 2015, 7, 1568915694.

12 R. I. Stefan-van Staden and J. F. van Staden, New Tool for Screening of Whole Blood for Early Detection of Breast Cancer Antigen (CA153), J. Mod. Med. Chem., 2013, 1, 86-91.

13 B. J. Sanghavi, O. S. Wolfbeis, T. Hirsch and N. S. Swami, Nanomaterial-based electrochemical sensing of neurological drugs and neurotransmitters, Microchim. Acta, 2015, 182(1-2), 1-41.

14 L. A. Gugoasa, R.-I. Stefan-van Staden, A. J. Muklive AlOgaidi, C. Stanciu-Gavan, J. Frederick van Staden, M.-C. Rosu and S. Pruneanu, Molecular Recognition of Colon Cancer Biomarkers: P53, KRAS and CEA in Whole Blood Samples, J. Electrochem. Soc., 2017, 164(9), B443-B447. 